ORIGINAL ARTICLE

\title{
Epididymal obstruction results in isolated sperm heads in post-vasectomy rats
}

\author{
Herkanwal S. Khaira, * Timothy G. Schuster, * Wen-Xiang Zhang, $\dagger$ Rodney Dunn, * Gary D. Smith*,,$\S$ \\ and Dana A. Ohl* \\ Departments of *Urology, †Oral and Maxillofacial Surgery, \$Obstetrics and Gynecology, and §Physiology, University of Michigan, Ann Arbor, MI, \\ USA
}

\begin{abstract}
Keywords:
epididymal obstruction, sterilization reversal, vasectomy, vasovasostomy

\section{Correspondence: \\ Timothy G. Schuster, Department of Urology, University of Michigan Medical Center, 3875 Taubman Center, 1500 East Medical Center Drive, Ann Arbor, Ml 48109-0330, USA. \\ E-mail: tgschust@med.umich.edu}

Received 1 March 2006; revised 15 July 2006; accepted 17 July 2006

doi:10.1111/j.1365-2605.2006.00709.x

\section{Summary}

The aim of this study was to determine if, following vasectomy, epididymal obstruction resulted in changes in vasal stump fluid using a rat vasectomy model. One hundred and twenty-two mature male rats underwent bilateral surgical vasectomy and subsequent unilateral epididymal obstruction. Animals were randomly assigned to one of the five cohorts, which determined the time to kill and vasal fluid assessment. Numbers of whole sperm and sperm heads were compared between the obstructed and non-obstructed sides. Parametric analysis of microscopic vasal fluid findings was performed using a paired $t$-test. Whole sperm and sperm heads were detected bilaterally among the initial five cohorts. On the obstructed epididymis side, percentage of whole sperm dropped from $36.9 \%$ to less than $1 \%$ and sperm heads increased from 63.2 to $99.7 \%$ at 12 weeks post-obstruction ( $p<0.05$ at each time interval). On the unobstructed side, percentage of whole sperm rose from 66.3 to $89.5 \%$ and sperm heads dropped from 33.7 to $10.5 \%$ ( $p<0.05$ at each time interval). At 12 weeks, the difference between the obstructed and non-obstructed sides for both percentage and quantity of whole sperm and heads was significant with a $p$ value of $<0.001$. In this rat model, following vasectomy and subsequent epididymal obstruction, testicular vasal stump fluid will contain progressively diminishing numbers of whole sperm and increases in the percentage and absolute numbers of sperm heads.

\section{Introduction}

Approximately 2-9\% of males undergoing vasectomy will undergo a subsequent reversal procedure (Fenster \& McLoughlin, 1981; Jequier, 1998; Heidenreich et al., 2000). The majority of these reversals are performed to restore fertility. The first-line reversal procedure is vasovasostomy, typically an end-to-end anastomosis of the ligated vasal segments. The success of this procedure is well documented, exceeding $90 \%$ under certain conditions (Silber, 1989; Belker et al., 1991). However a vasectomy reversal will not achieve patency if a co-existing epididymal obstruction is not recognized and bypassed.

The presence of epididymal obstruction occasionally complicates vasectomy reversal. It is theorized that chronic backpressure from the obstructed vasal segment is transmitted to the epididymal tubules (Silber, 1981;
McDonald, 1996). The thin-walled epididymal tubules unable to withstand the increased hydrostatic pressure dilate and may on occasion rupture. The subsequent inflammatory reaction, driven, in part, by an immune response to the haploid germinal cells, often results in localized granuloma formation with subsequent fibrosis and obstruction (Kennedey \& Heidger, 1980).

In the event of a complete epididymal obstruction, intact viable spermatozoa will not be identified downstream of the occlusion. However, the absence of intact spermatozoa in the vas deferens does not absolutely indicate epididymal obstruction; temporary or permanent azospermia may follow vasectomy as a result of inflammatory changes, sperm degradation or vascular insults (Flickinger, 1982; Flickinger et al., 1995).

During vasectomy reversal, fluid appearance, microscopic evaluation and gross examination of the epididy- 
mis and vas deferens have been categorized and graded to determine the likelihood of systemic patency (Belker et al., 1991). At the time of surgery, the presence of sperm within the vasal fluid is an indication to proceed with vasovasostomy. It is more controversial if one should perform vasovasostomy or vasoepididymostomy when isolated sperm heads are found. Commonly, the absence of spermatozoa from the bilateral vas is associated with a $31 \%$ pregnancy rate following vasovasostomy (Belker et al., 1991). However, no comprehensive evaluation of vasal fluid following the creation of a secondary blockage within the epididymis after vasectomy has been reported to our knowledge. We sought to better define the immediate and short-term microscopic changes in the testicular vasal fluid that occurred following vasectomy and subsequent epididymal obstruction, through use of a randomized animal model cohort.

\section{Material and methods}

The investigation protocol was approved by the University Committee for the Use and Care of Animals. One hundred and twenty-two sexually mature male SpragueDawley rats were obtained from the Charles River Laboratories (Wilmington, MA, USA). On day 0, under general anaesthesia, all animals underwent bilateral vasectomy. Anaesthesia consisted of $80 \mathrm{mg} / \mathrm{kg}$ intramuscular Ketamine and $10 \mathrm{mg} / \mathrm{kg}$ intramuscular Xylazine. Adequate depth of anaesthesia was confirmed prior to proceeding with surgical incision. All surgeries were performed by one of two surgeons, utilizing an operative microscope.

Vasectomy was performed through $2.5-\mathrm{cm}$ paramedian scrotal incision. The tunica vaginalis was opened and the testes and epididymis were delivered. The vasal artery was dissected free from the vas deferens. The integrity of the vasal artery was maintained in all animals. The vas was ligated with 4-0 non-absorbable suture at $1.5 \mathrm{~cm}$ from the vasal-epididymal junction and at the distal stump. The vas was then transected and a $0.5-\mathrm{cm}$ segment was removed. The scrotal contents were returned to their anatomic location within the tunic vaginalis. The skin was then closed in interrupted fashion. The identical procedure was performed on the contralateral hemiscrotum. During week 4, all animals underwent unilateral iatrogenic epididymal obstruction. This procedure was performed under systemic anaesthesia as previously detailed. A 1-cm longitudinal incision was made on the paramedian mid-scrotum. The epididymis was generally identified with minimal dissection and the mid-corpora of the epididymis was ligated with 6-0 non-absorbable suture. The epididymis was consistently obstructed in this manner in the same location for all the subject animals. The side of epididymal obstruction (i.e. left vs. right) was randomly predetermined. A contralateral sham operation was not performed to minimize the possibility of inadvertent injury to the control side epididymis.

During weeks 5, 6, 8, 12 and 16 (post-epididymal obstruction weeks $1,2,4,8,12), 14-24$ rats in the randomly assigned cohorts were respectively killed. Animals were killed at room temperature by $\mathrm{CO}_{2}$ asphyxiation. The scrotal contents were removed intact. The obstructed vasal end was opened and vasal fluid was assessed grossly. The vas was gently milked from the level of the vaso-epididymal junction to the transected end to express and quantify all fluid. Invariably, repeating this procedure resulted in expression of some fluid. There was no blood contamination of the vasal fluid during this step that affected sperm evaluation.

A single investigator assessed the obstructed and nonobstructed side vasal fluid. Vasal fluid was diluted in $20 \mu \mathrm{L}$ of Hepes-buffered solution with $0.2 \%$ bovine serum albumin at $37^{\circ} \mathrm{C}$. Manual quantification of vasal fluid contents was performed with a haemocytometer; this included number of sperm heads (without bodies), sperm tails (without heads), whole sperm and motility.

\section{Statistical methods}

The numbers of sperm heads at each of the two sites of vasal fluid (obstructed epididymis and non-obstructed epididymis) were determined at weeks $1,2,4,8$ and 12 after undergoing the unilateral epididymal obstruction. The obstructed epididymis and non-obstructed epididymis were compared using a paired $t$-test for each site/ week combination. To assess the trend over time, a mixed model was produced separately for each site. Each model initially contained a main effect for week and an indicator for obstructed epididymis vs. unobstructed epididymis, plus an interaction between week and obstruction indicator. This indicator was used to assess whether the slope of the count over time was different between the obstructed and unobstructed groups. A backwards model building process was then used to arrive at the most parsimonious model. The 5\% significance level was applied to all tests and models, and all analyses were performed using the SAS System (Cary, NC, USA).

\section{Results}

One hundred and twenty-two rats were initially entered into the study protocol. Twenty-four rats were prospectively randomly assigned to each cohort (1-5), which corresponded with date of killing following epididymal occlusion. Ultimately, 24 rats were in cohort 1 (week 1), 23 in cohort 2 (week 2), 24 in cohort 3 (week 4), 20 in cohort 4 (week 8) and 14 in cohort 5 (week 12). Two rats 
were isolated from the remaining population because of suspected viral infection and were determined healthy for inclusion and killed at week 22 as a sixth cohort. There were 15 mortalities prior to completion of the protocol, primarily from anaesthetic-related complications and these account for the excluded animals.

Among early findings, vasal sperm granulomas were found in over $98 \%$ of all ligated vasa. All but two (1.9\%) of the epididymal obstructed sides also demonstrated epididymal sperm granulomas.

Figure 1 demonstrates the absolute number of isolated sperm heads and whole sperm by cohort. The difference between the non-obstructed epididymis and obstructed epididymis side for both absolute number and per cent number of sperm heads and whole sperm demonstrated a $p$-value of $<0.05$ at each time point. Motility rates were less than $10 \%$ in each group and did not differ significantly.

In cohort 5 (12 weeks post-epididymal obstruction), all but two of 14 rats demonstrated either isolated sperm heads or no sperm components in the obstructed epididymis side vasal stump. The epididymal obstructed side after 12 weeks had significantly higher levels of isolated heads than non-obstructed side $(p<0.001)$. On the unobstructed epididymis side at 12 weeks, one of 14 animals was found to have 'heads only.' After 22 weeks there was no whole sperm found on the obstructed side. Statistical analysis was not performed on this group because of the small size of the cohort.

Table 1 lists the percentages of isolated sperm heads and whole sperm separated by cohort. Among the obstructed epididymis subgroup, per cent of isolated sperm heads increased with time from epididymal occlusion, conversely whole sperm numbers decreased with each interval. The differences between the unobstructed epididymis and obstructed epididymis side in terms of whole sperm and sperm heads were significant at each time point with a $p$-value $<0.05$.
Table 1 Per cent number of whole sperm and sperm heads

Week 1 Week 2 Week 4 Week 8 Week 12

\begin{tabular}{|c|c|c|c|c|c|}
\hline \multicolumn{6}{|l|}{ Isolated sperm heads } \\
\hline Control side (\%) & 33.7 & 33.9 & 14.4 & 17.2 & 10.5 \\
\hline $\begin{array}{l}\text { Epididymal } \\
\text { obstruction (\%) }\end{array}$ & 63.2 & 68.7 & 89.3 & 95.0 & 99.7 \\
\hline$p$-value & 0.0375 & 0.0017 & $<0.001$ & $<0.0001$ & $<0.001$ \\
\hline \multicolumn{6}{|l|}{ Whole sperm } \\
\hline Control side (\%) & 66.3 & 66.1 & 85.6 & 82.8 & 89.5 \\
\hline $\begin{array}{l}\text { Epididymal } \\
\text { obstruction (\%) }\end{array}$ & 36.9 & 31.3 & 10.8 & 5.0 & 0.3 \\
\hline$p$-value & 0.0329 & 0.0039 & $<0.001$ & $<0.001$ & $<0.001$ \\
\hline
\end{tabular}

\section{Discussion}

In this rodent model, unilateral iatrogenic epididymal obstruction following bilateral vasal occlusion demonstrated significant differences within the microscopic characteristics of the vasal stump fluid. Notably, absolute numbers and per cent quantity of whole sperm decreased over each time point among the rats with subsequent epididymal obstruction. On the non-obstructed epididymis side, whole sperm percentages ranged between 66.1 and $89.5 \%$ over the 12 weeks post-epididymal obstruction. This finding implies that whole sperm, when isolated within the segment distal to the epididymal obstruction, begins the process of degeneration almost immediately. Decapitation of these progressively degenerating sperm, would thereby account for the increasing per cent and absolute numbers of sperm heads concurrent with the decreasing numbers of whole sperm.

Although not quantified, epididymal granulomas were rarely found on the unobstructed epididymis side and often correlated with difficulty identifying any sperm in the vasal segment. This finding indicated that vasal sperm in rats with non-iatrogenic epididymal obstructions, as

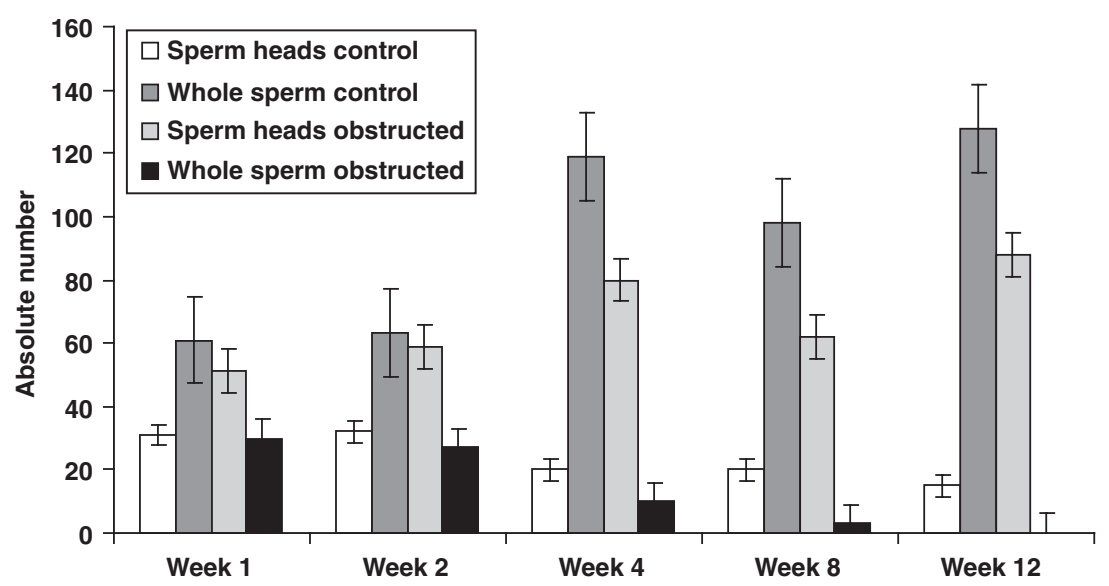

Figure 1 Absolute numbers of whole sperm and sperm heads over time (including standard error of mean averaged per subject). 
suspected by the presence of an epididymal granuloma, followed a similar degradation pattern to the iatrogenic group.

Micro-anatomical evaluations of post-obstructive vasal fluid in the literature are limited; these are primarily veterinary studies that were reported in prior decades. Paufler \& Foote (1968) assessed post-obstructive changes in epididymal and vasal fluid in a rabbit model. They found increasing numbers of detached heads downstream among rats with epididymal obstruction. In this experiment, among rats with isolated vasal occlusion, sperm at the vasa was relatively normal. Gaddum \& Glover (1965) found epididymal obstruction was associated with an early increase in sperm motility proximal to the obstruction followed by degeneration of the sperm. Glover (1969) found that subsequent to occlusion of the rabbit epididymis, an increase in degenerated sperm was seen above level of ligation after 3 weeks.

The degeneration of spermatozoa in the isolated epididymal cauda and vas stump is likely the result of changes in the luminal fluid following obstruction. This would include activation of hydrolytic enzymes because of the obstruction (Jones, 2004). The persistence of sperm heads in this environment relates to the condensed nature of chromatin and the sturdy nature of the perinuclear theca (Powell et al., 1990).

Belker et al. (1991) assessed intraoperative vasal fluid characteristics during vasectomy reversal and correlated these findings with patency and fecundity rates. The microscopic finding of 'only heads without tails' was associated with a $75.3 \%$ patency rate postoperatively. Additionally, the microscopic absence of any sperm or sperm components was associated with a $60.2 \%$ patency rate. They concluded that absence of sperm or sperm components does not absolutely indicate epididymal obstruction. However, lengthy intervals of obstruction and non-watery fluid in conjunction with this finding increased the likelihood of concurrent epididymal obstruction. Recently, Sigman (2004) noted patency rates approaching $100 \%$ following vasovasostomy when sperm parts were found in vasal fluid during vasectomy reversal. These investigations suggest that finding sperm heads only during vasectomy reversal may actually indicate an absence of epididymal occlusion among human subjects. Our study of rat subjects indicates that in the early period ( $<12$ weeks) following vasectomy and subsequent epididymal obstruction, finding sperm heads only is more common on the epididymal-obstructed side. However, the long-term changes in the vasal fluid were not assessed and in fact sperm heads only could potentially be found among non-epididymal obstructed rats; the aim of this study was not to characterize the long- term changes, but instead to determine qualities associated with recent epididymal obstruction following vasectomy.

A limitation of our investigation is the use of an animal model. Despite considerable similarities between the rat and human gonad, epididymis and vas deferens, these results cannot be directly applicable for decisionmaking or treatment algorithms in human subjects. The rat epididymis is quite fragile and develops granulomas more frequently than the human epididymis (Miller et al., 1984; McDonald, 1996; McGinn et al., 2000). The degradation of sperm isolated in the rat vas may not entirely parallel breakdown in the human; although both species' sperm have resilient head pieces, the process and time course to decapitation may not be similar. Additionally, although this investigation demonstrated a progressive, short-term increase in sperm decapitation among post-vasectomy, epididymal obstructed rats compared with post-vasectomy rats with patent epididimides, the long-term (i.e. years) changes in vasal fluid following vasectomy were not assessed. Potentially, decapitation could affect all post-vasectomy vasal sperm, independent of epididymal patency. However, it should be noted that concurrent with the finding of increasing numbers of sperm heads was the reduction in the absolute number of whole sperm among the subjects with epididymal obstruction. Ultimately, only four $(0.3 \%)$ whole sperm were found on the epididymal obstruction side among the 14 rats in cohort 5 (week 12), on the control side 1797 (89.5\%) whole sperm were counted. This finding indicates that the presence of whole sperm reliably predicts epididymal patency, and in turn the absence of whole sperm points more towards epididymal obstruction. Further investigations including long-term animal studies and human follow-up studies will better determine if the finding of isolated sperm heads is a durable characteristic of post-vasectomy epididymal obstruction.

\section{Conclusions}

In this controlled, randomized investigation, rat vasal stump fluid after vasectomy was compared with post-vasectomy vasal fluid following epididymal occlusion to find that epididymal obstruction in post-vasectomy rats resulted in progressively lower numbers of whole sperm in the testicular vas deferens. Concurrently, the per cent of isolated sperm heads rose following epididymal obstruction. These finding indicated that in the rat model isolated sperm heads found in vasal stump fluid following vasectomy may be related to recent epididymal obstruction. Further long-term investigations are needed to determine the durability of this finding. 


\section{References}

Belker, A. M., Thomas, A. J. Jr, Fuchs, E. F., Konnak, J. W. \& Sharlip, I. D. (1991) Results of 1,469 microsurgical vasectomy reversals by the Vasovasostomy Study Group. Journal of Urology 145, 505-511.

Fenster, H. \& McLoughlin, M. G. (1981) Vasovasostomy microscopy versus macroscopic techniques. Archives of Andrology 7, 201-204.

Flickinger, C. J. (1982) The fate of sperm after vasectomy in the hamster. Anatomical Record 202, 231-239.

Flickinger, C. J., Howards, S. S. \& Herr, J. C. (1995) Effects of vasectomy on the epididymis. Microscopy Research and Technique 30, 82-100.

Gaddum, P. \& Glover, T. D. (1965) Some reactions of rabbit spermatozoa to ligation of the epididymis. Journal of Reproduction \& Fertility 9, 119.

Glover, T. D. (1969) Some aspects of function in the epididymis. Experimental occlusion of the epididymis in the rabbit. International Journal of Fertility 14, 216-221.

Heidenreich, A., Altmann, P. \& Engelmann, U. H. (2000) Microsurgical vasovasostomy versus microsurgical epididymal sperm aspiration/testicular extraction of sperm combined with intracytoplasmic sperm injection. A cost benefit analysis. European Urology 37, 609-614.

Jequier, A. M. (1998) Vasectomy related infertility: a major and costly medical problem. Human Reproduction 13, 17571759.

Jones, R. (2004) Sperm survival versus degradation in the mammalian epididymis: a hypothesis. Biology of Reproduction 71, 1405-1411.
Kennedey, S. W. \& Heidger, P. M. (1980) Fine structure of the spermatic granuloma of the rat vas deferens following vasectomy. Anatomical Record 198, 461-474.

McDonald, S. W. (1996) Vasectomy review: sequalae in the human epididymis and ductus deferens. Clinical Anatomy 9, 337-342.

McGinn, J. S., Sim, I., Bennett, N. K. \& McDonald, S. W. (2000) Observations on multiple sperm granulomas in the rat epididymis following vasectomy. Clinical Anatomy 13, 185-194.

Miller, R. J., Killian, G. J. \& Vasilenko, P. (1984) Effects of long- and short term vasectomy on structural and functional parameters of the rat. Journal of Andrology 5, 381-388.

Paufler, S. K. \& Foote, R. H. (1968) Morphology, motility and fertility of spermatozoa recovered from different areas of ligated rabbit epididymides. Journal of Reproduction \& Fertility 17, 125-137.

Powell, D., Cran, D. G., Jennings, C. \& Jones, R. (1990) Spatial organization of repetitive DNA sequences in the bovine sperm nucleus. Journal of Cell Science 97, 185-191.

Sigman, M. (2004) The relationship between intravasal sperm quality and patency rates after vasovasostomy. Journal of Urology 171, 307-309.

Silber, S. J. (1981) Reversal of vasectomy and the treatment of male infertility: role of microsurgery, vasoepididymostomy, and pressure induced changes in vasectomy. Urologic Clinics of North America 8, 53-62.

Silber, S. J. (1989) Pregnancy after vasovasostomy for vasectomy reversal: a study of factors affecting long-term return of fertility in 282 patients followed for 10 years. Human Reproduction 4, 318-322. 